\title{
Introduction : l'évolution européenne des dépôts et de leurs ateliers
}

Introduction: the European evolution of depots and their workshops

Jean Metz et Yves Machefert-Tassin

\section{OpenEdition}

\section{Journals}

Édition électronique

URL : https://journals.openedition.org/rhcf/1771

DOI : 10.4000/rhcf.1771

\section{Éditeur}

Rails \& histoire

\section{Édition imprimée}

Date de publication : 1 décembre 2003

Pagination : $375-389$

ISBN : 0996-9403

ISSN : 0996-9403

\section{Référence électronique}

Jean Metz et Yves Machefert-Tassin, «Introduction : l'évolution européenne des dépôts et de leurs ateliers », Revue d'histoire des chemins de fer [En ligne], 28-29 | 2003, mis en ligne le 29 décembre 2014, consulté le 22 avril 2022. URL : http://journals.openedition.org/rhcf/1771 ; DOI : https://doi.org/ $10.4000 /$ rhcf. 1771 


\section{Introduction : l'évolution européenne des dépôts et de leurs ateliers}

La Revue d'histoire des chemins de fer, en son numéro 18 (printemps 1998), a présenté globalement l'évolution des dépôts français de matériel moteur et des ateliers correspondants, sur 160 ans, avec une division périodique jalonnée approximativement par les années 1900 et 1973. Cette division correspondait davantage, pour le tournant du siècle, à la variation de leur espacement géographique accru par les parcours en ligne des locomotives. D'où le début d'une concentration en établissements moins nombreux et de plus en plus chargés en nombre de machines et d'hommes, pour qu'ils puissent assurer dans des ateliers, qui regroupaient parfois jusqu'à la moitié des effectifs, les opérations de gros entretien et de réparation locale. Il est ensuite notable que, jusque vers les années 1970 et malgré l'apparition depuis 1925-1930 des modes de traction électrique, puis des autorails, ces coexistences n'aient pas amené systématiquement des remaniements fondamentaux, ni des spécialisations par établissement (sauf pour les banlieues des capitales), autres que des aménagements locaux particuliers, généralement dans les bâtiments anciens, avec remaniement des voies d'accès.

Par contre, avec la disparition ultérieure de la traction vapeur en faveur de l'électrique et du Diesel, puis le retour massif aux conceptions de rames indéformables, ou à segments facilement " coupables », que ce soit en transport à grande vitesse ou en dessertes régionales, les installations existantes ont été profondément modifiées, ou reconstruites, et pas seulement dans le plan des voies remanié lors de la disparition des grandes surfaces nécessaires aux charbons de la vapeur, ou l'affectation des bâtiments. Or, on constate dans toute l'Europe une évolution assez analogue, quel que soit le réseau considéré.

En introduction à cette séance, fournie en exemples très divers de l'évolution d'abord anglaise puis française des établissements parmi les plus typiques dès les tout premiers débuts de la traction à vapeur, je voudrais montrer comment, à l'échelle européenne, nos voisins, avec des variantes locales, ont finalement suivi une démarche moins éloignée de la nôtre qu'il n'y paraîtrait (et telle que l'on peut le constater de nos jours sur l'ensemble de l'Union européenne), en se limitant au seul aspect architectural des bâtiments principaux de dépôts (fig. 1). 


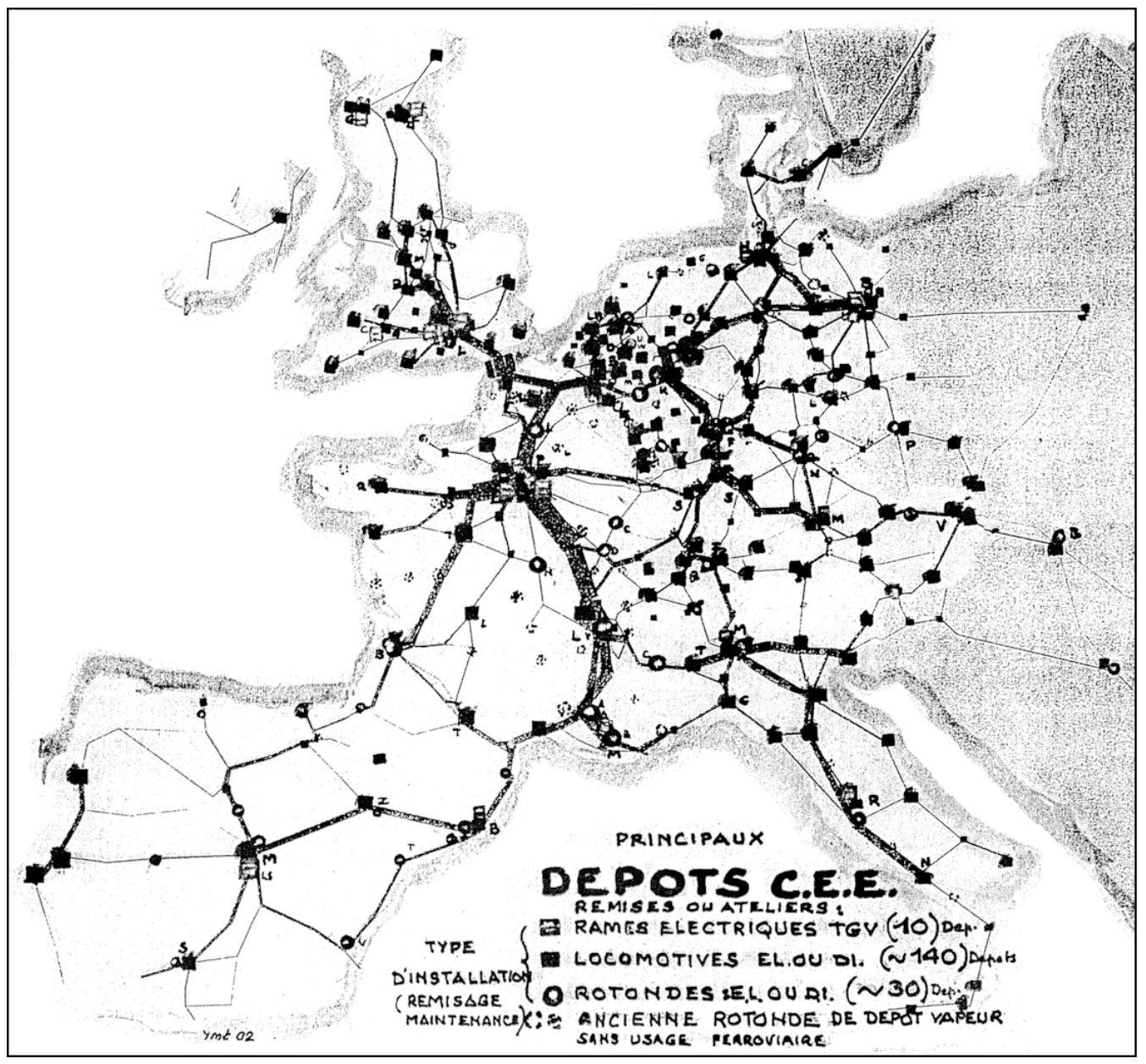

Figure 1. Types d'installations principales de dépôts dans l'Union européenne. Remises à voies parallèles ou en rotonde pour locomotives ou pour rames et rotondes encore existantes sans usage ferroviaire autre que musée ou atelier voie. Dessin Y. Machefert-Tassin. 
Depuis l'origine des chemins de fer, rappelons que deux types principaux de bâtiments ont coexisté sous des formes diverses de disposition des voies et de leur accès : rotondes avec plaque ou pont tournant, couvert ou non, d'où les remises annulaires, et remises carrées ou rectangulaires avec des appareils de desserte des trois modèles : plaques, ponts transbordeurs ou aiguillages. Elles couvrent alors des voies soit en étoile, soit parallèles. Après le hangar simple des tous débuts le modèle en rotonde complète semble bien remonter aux années 1840 et être le plus répandu à partir de la Grande-Bretagne. Carré ou rond et multipliable, quelques exemples évolutifs l'illustrent (fig. 2 et fig. 3) tel Paddington-GWR. Elles se retrouvent vite sur le continent (à coupoles ou anneaux) de la France à la Russie (fig. 4) après 1840. Celles à voies parallèles les suivent de peu, avec l'accroissement très rapide de la longueur des machines, d'abord avec quelques voies desservies par aiguillages, puis beaucoup plus nombreuses pour les grands dépôts avec des voies en cul-de-sac pour l'atelier (Belgique, Hollande, Italie) (fig. 5 et fig. 6) ou à chariots transbordeurs, couverts puis découverts (Allemagne, Suisse, France) (fig. 7, fig. 8 et fig. 9). Ceci, notons-le dès à présent, restera valable quel que soit le mode de traction, vapeur, puis électrique et Diesel. Notons au passage que la Grande-Bretagne pratiqua aussi bien les constructions carrées ou rectangulaires de remisage sur des voies en étoile, avec 24 à 28 fosses desservies par plaques, et 90 à $100 \mathrm{~m}^{2}$ par place couverte, qu'avec 12 voies parallèles, et 48 à 58 places, sur fosses en long, nécessitant seulement $70 \mathrm{~m}^{2}$ par machine. Dès 1860 et jusqu'en 1910 on trouve ainsi des «quadratures de rotonde » souvent accolées par deux ou quatre remises, même six, offrant jusqu'à 112 places à couvert sur un hectare par exemple à Newcastle (NER) et Hull-Dairycoates (NE) ou Swindon et Old Oak Common (GWR) (fig. 3, fig. 10 et fig. 11). Vu l'évolution en longueur des machines, la faveur revient ensuite aux voies parallèles mais, curieusement et seulement au Royaume-Uni, ces rotondes sous couvert, carrées, seront les premières à pouvoir être facilement transformées en remises à voies parallèles, jusqu'à 25 voies (Rugby, LMR) et 100 machines, ce qui ne sera jamais possible avec les autres modèles de rotondes ou remises annulaires, abandonnées avant 1940 à peu près partout, sauf en France à cause de l'influence tenace du PLM, la région Sud-Est devenant responsable des nouveaux projets unifiés de dépôts. Ces grandes remises à voies parallèles seront ensuite facilement adaptées aux modes de traction électrique ou Diesel et, après 1965, certains dépôts anglais (Toton, Newton Heath), allemands ou néerlandais atteignent 15 à 17 voies parallèles desservies par aiguillages. Ils sont alors soit en tiroir (deadend), soit traversants, soit avec un transbordeur médian ou d'extrémité (voir schémas de voies suisses ou allemands, fig. 7 et fig. 8). 

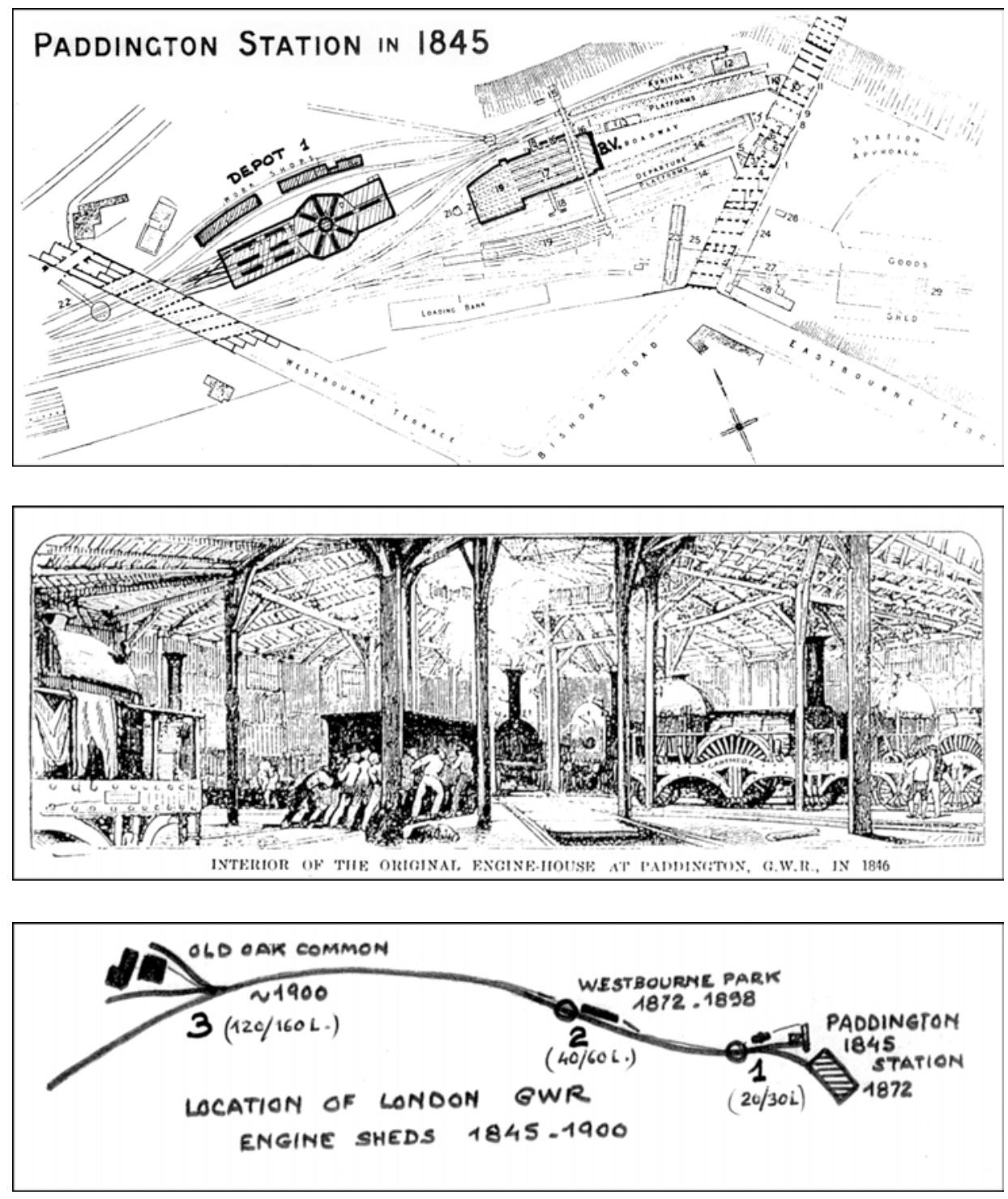

Figure 2. L'évolution des différents dépôts britanniques selon le modèle du Great-Western à Paddington avec trois lieux successifs convenant à des nombres croissants de locomotives de 1845 à 1900, pour loger 20, 40 puis 120 engins. Dessins Y. Machefert-Tassin. Au centre, l'intérieur de la première rotonde de 1838 à 1852 avec locomotives à voie large. Source : Great Western Trust. 


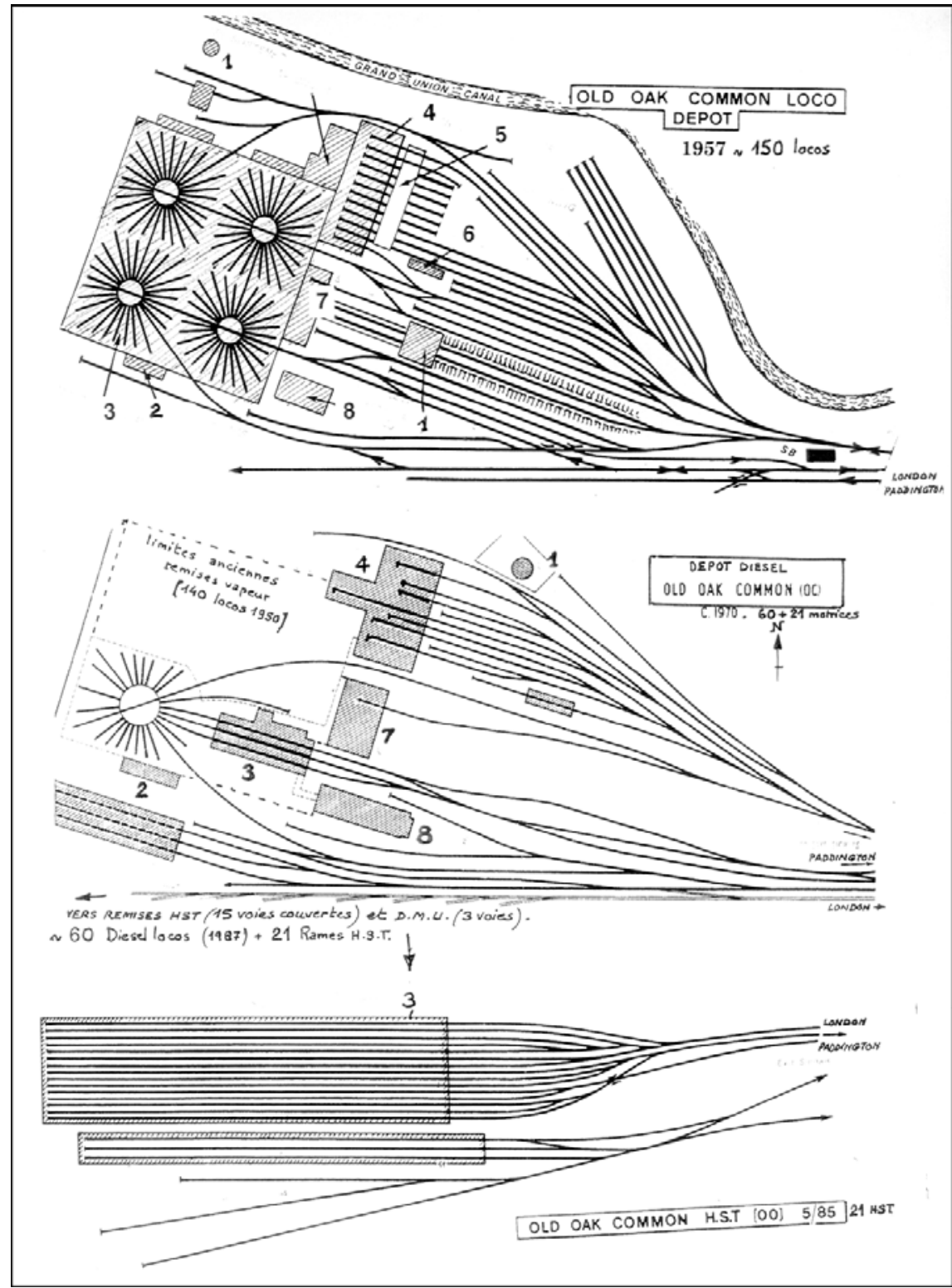

Figure 3. Le même exemple, Old Oak Common, avec son évolution ultérieure de 1900 à nos jours. De haut en bas : les quatre rotondes et l'atelier à transbordeur (vapeur, 1957) puis l'adaptation au Diesel (locomotives et autorails, 1970) enfin, plus au Sud, la nouvelle remise des trains Diesel à $200 \mathrm{~km} / \mathrm{h}$ (HST de 1985 à nos jours). Dessins Y. Machefert-Tassin d'après des documents fournis par le Great Western Railway. 


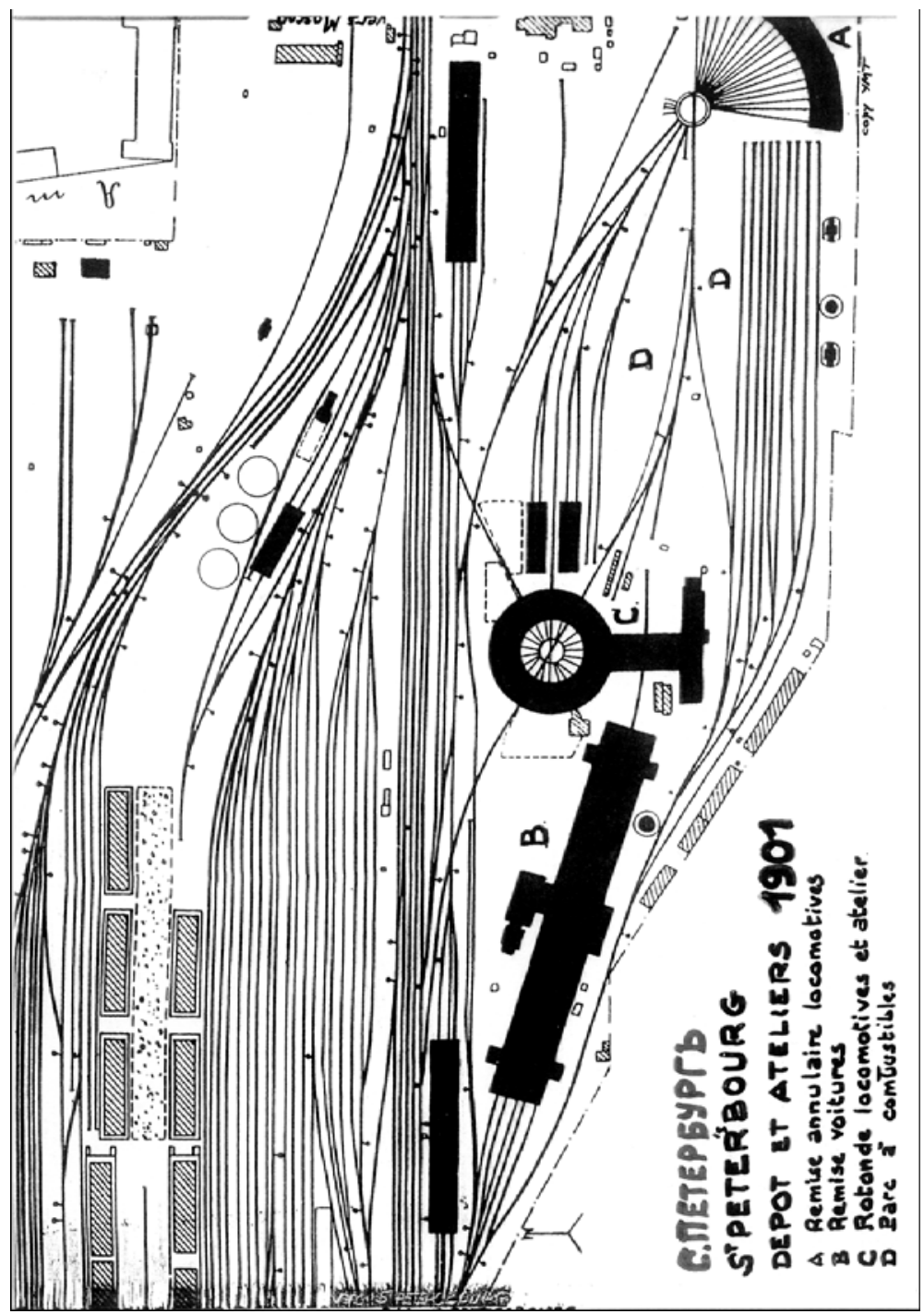

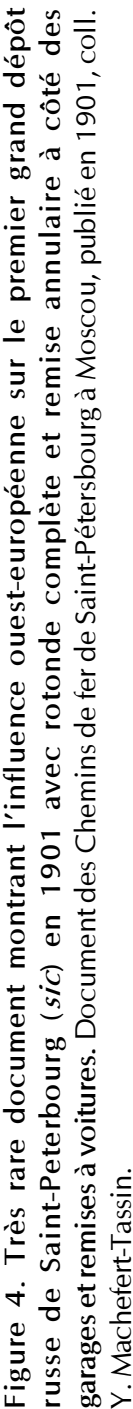




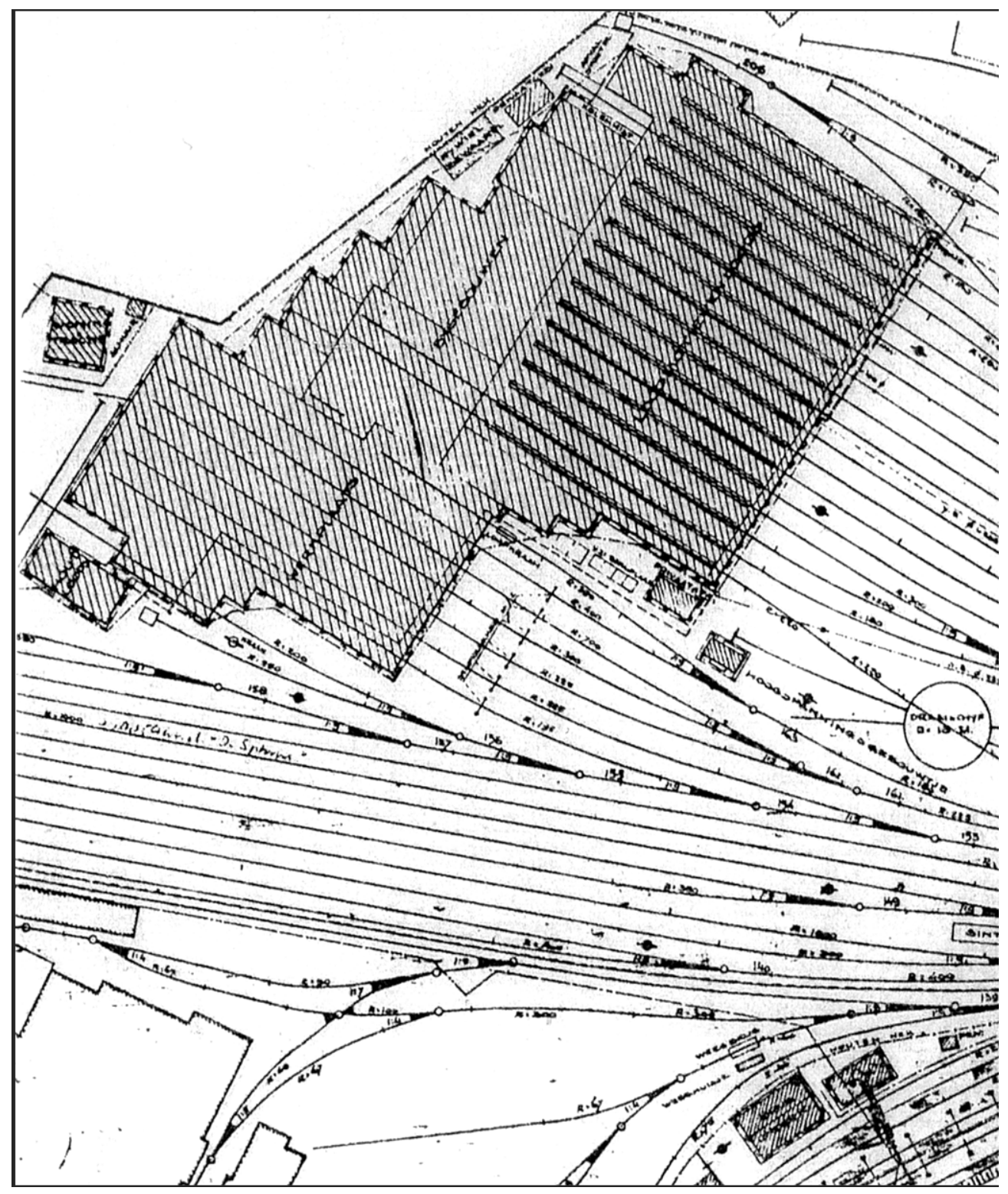

Figure 5. Un des plus grands dépôts néerlandais converti très directement et simplement de la vapeur à l'électrique : celui de Maastricht. Cl. Nederlandse Spoorwegen, coll. Y. Machefert-Tassin. 


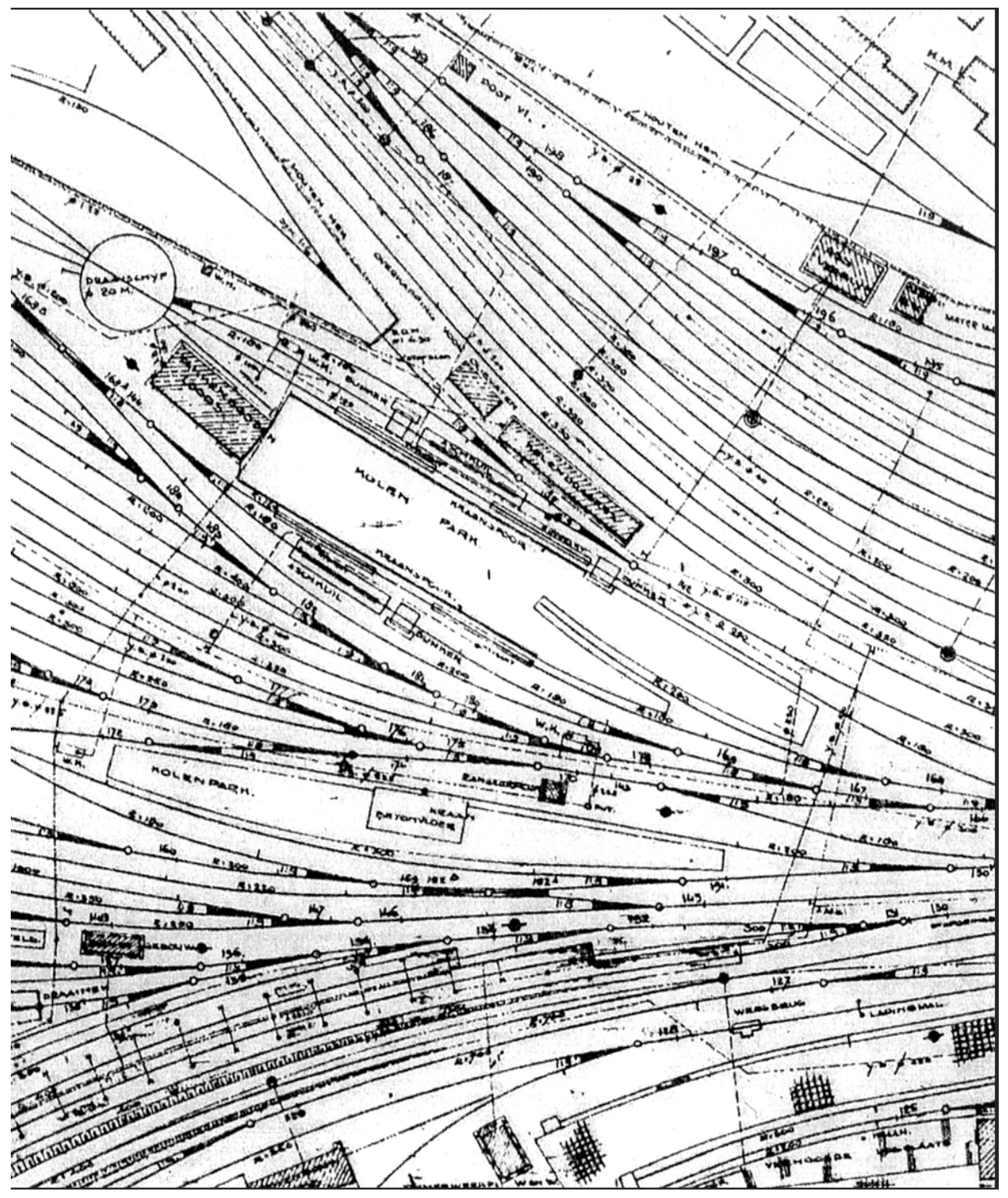




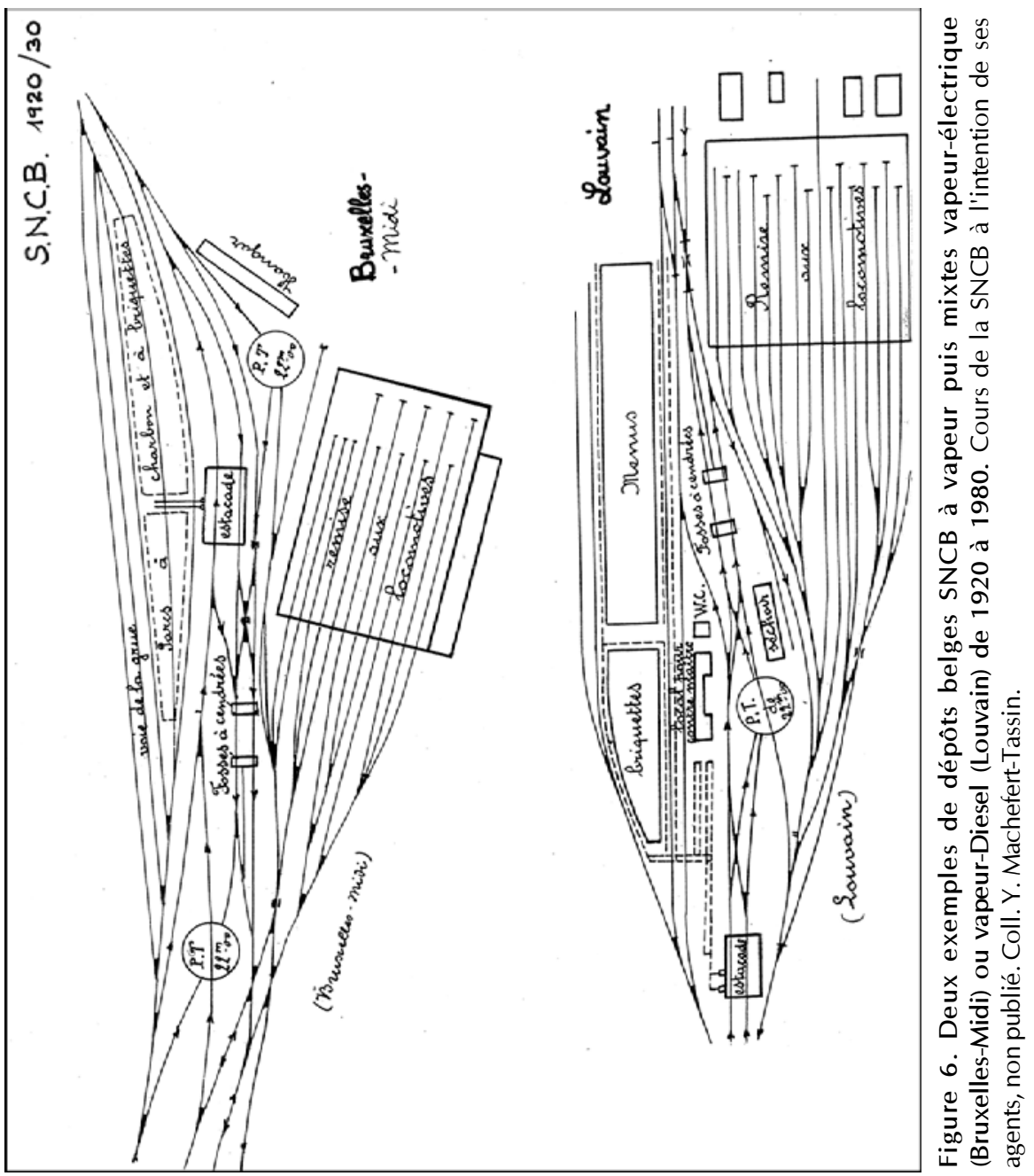




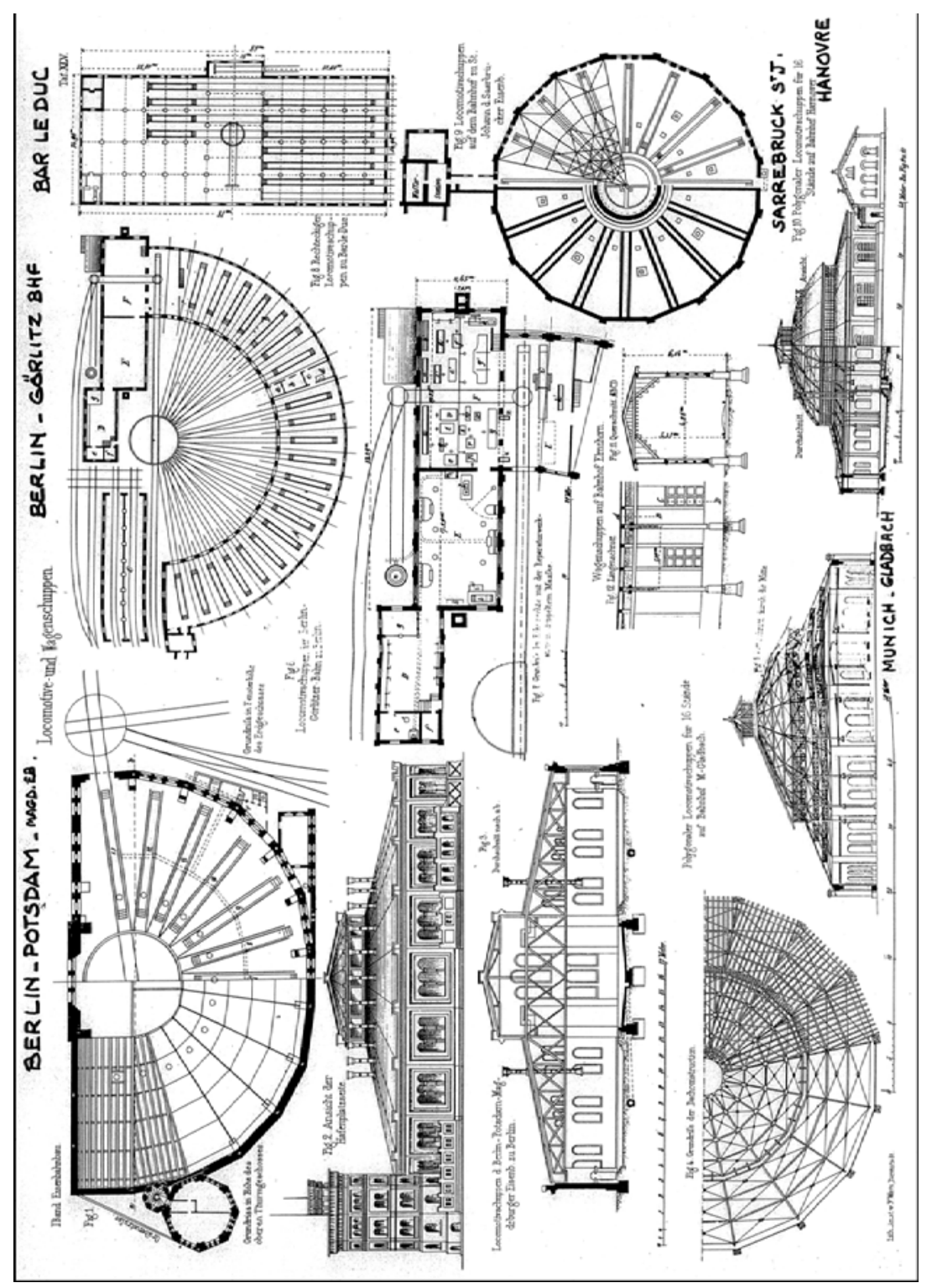

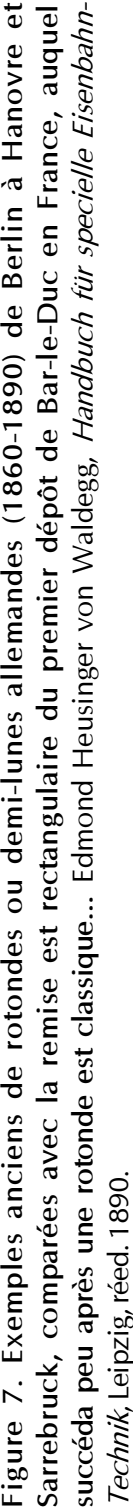




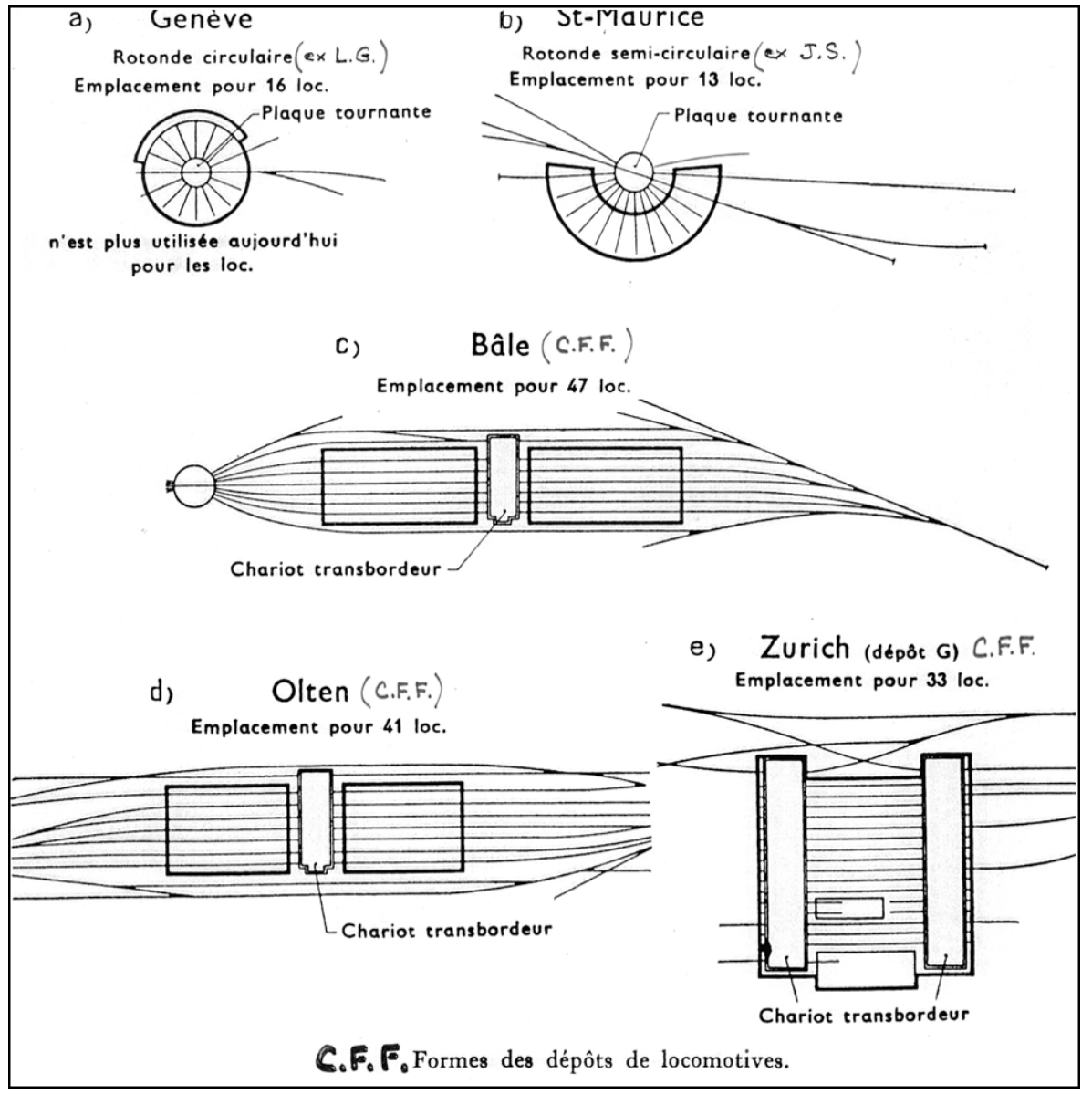

Figure 8. Exemples des cinq principes réalisés en Suisse pour des dépôts à vapeur, puis électriques, de 1860 à 1930, puis à 47 places couvertes. Pour I'histoire, la rotonde de Genève du Lyon-Genève d'origine est aujourd'hui (2001) remplacée, vers Sécheron, par la longue remise-atelier des rames pendulaires ICN à $200 \mathrm{~km} / \mathrm{h}$. Cours des Chemins vde fer férdéreaux suisses, 1930. Coll. Y. Machefert-Tassin. 


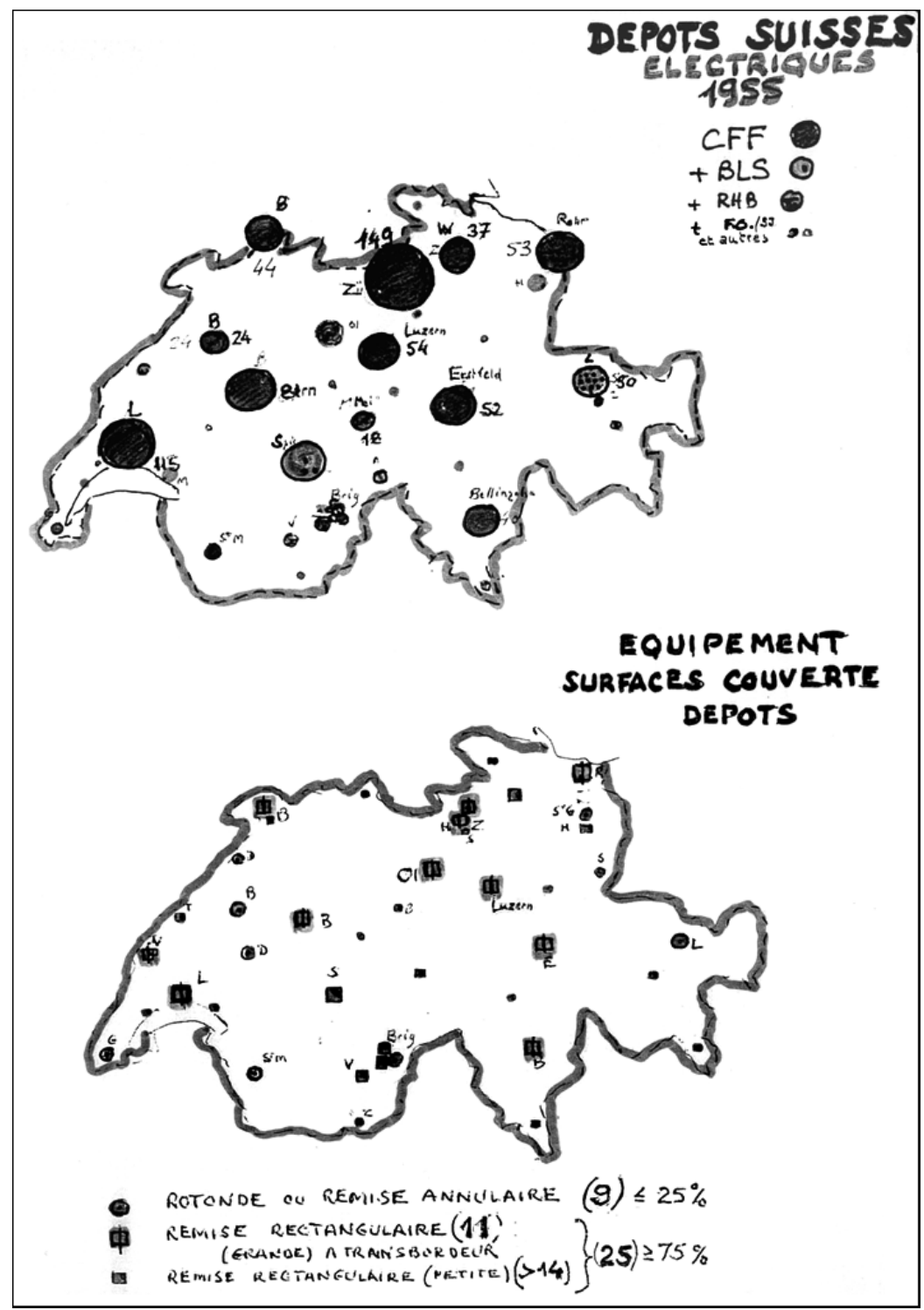

Figure 9. Sites des principaux dépôts suisses : importance en nombre d'engins électriques (1955) et type d'équipement des surfaces couvertes, soit un quart en rotonde ou anneau, et trois quarts en remises à voies parallèles. Dessin Yves Machefert-Tassin d'après des documents fournis par les réseaux concernés. 


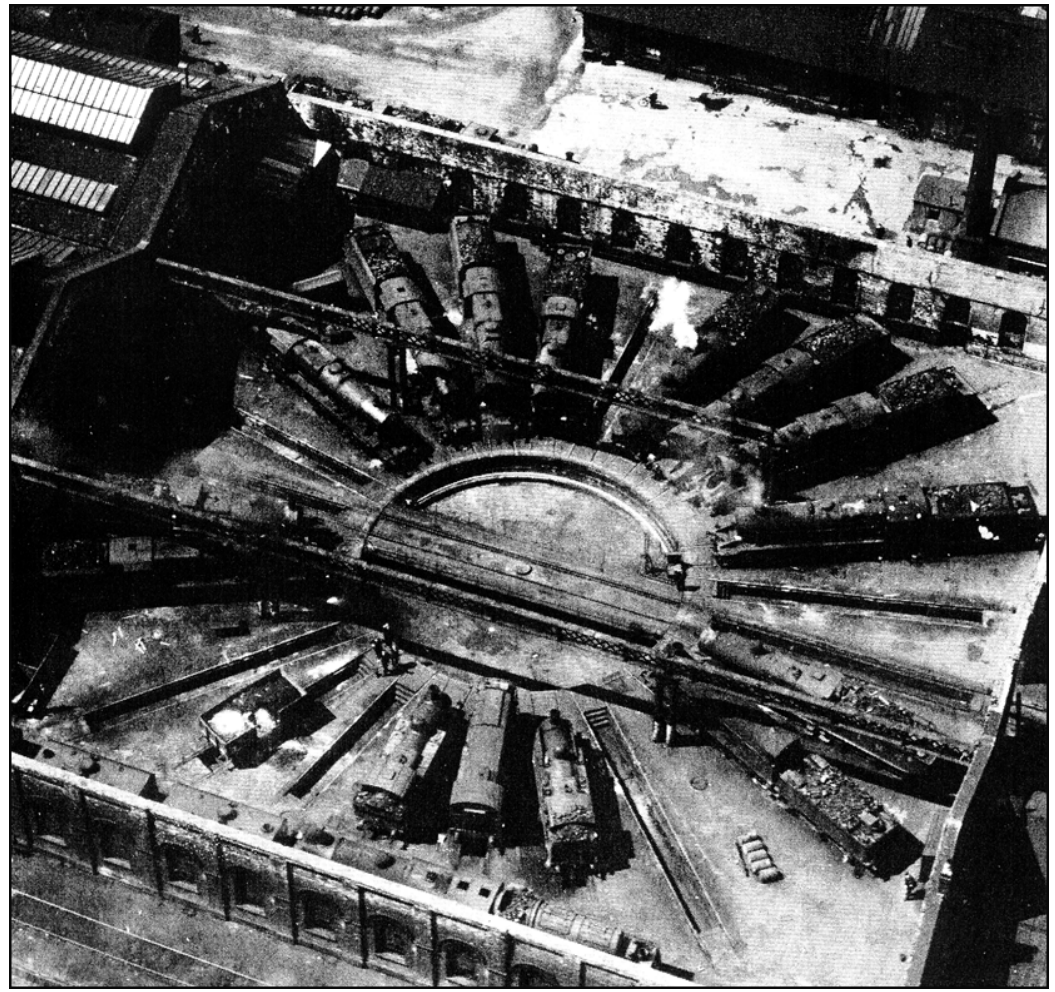

Figure 10. Cette remarquable vue aérienne de la rotonde de Toton (British Railways) où manque la toiture après la guerre (1950) montre par les portiques supports et les murs la facilité ultérieure de conversion aux tractions modernes. Vers 1970 ce sera cependant une nouvelle remise à voies parallèles qui sera construite. $\mathrm{Cl}$. British Railways, coll. Y. MachefertTassin. 


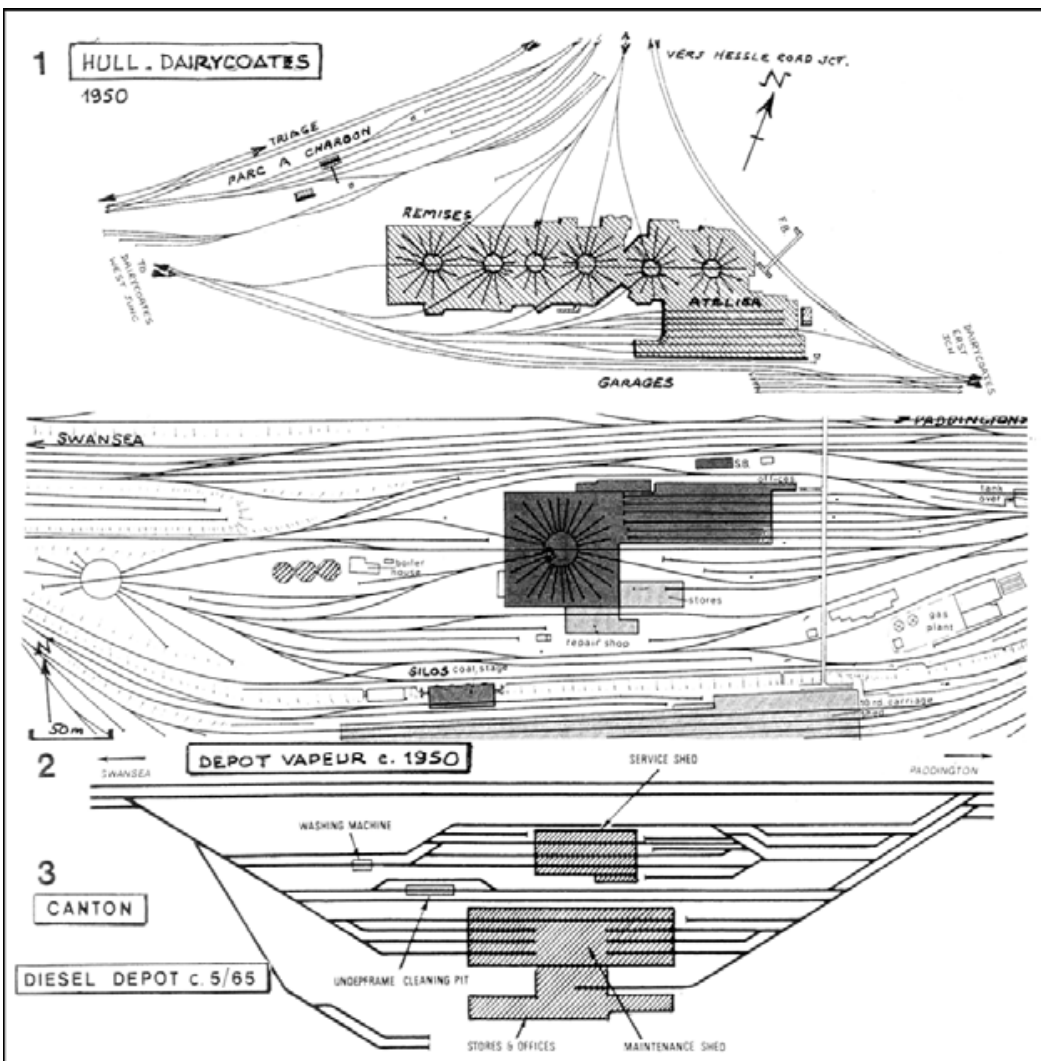

(87)

121 D.H. locos

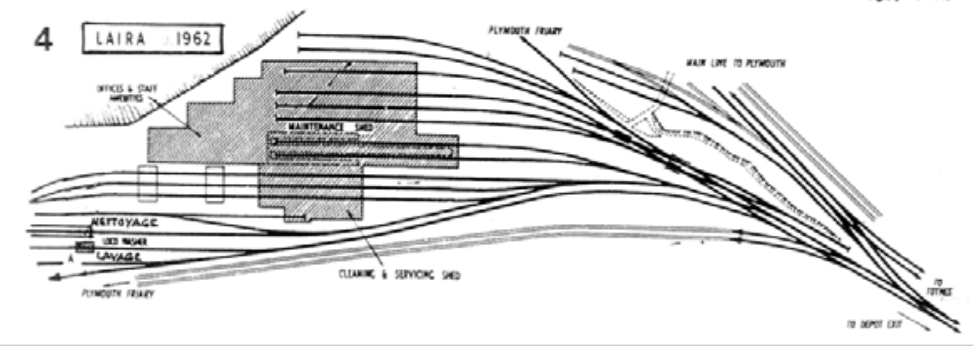

Figure 11. Trois autres exemples britanniques de conception puis d'adaptation de la vapeur au Diesel, ou disparition (Hull-Dairycoates à six rotondes, fermé en 1960), reconstruction (Canton, près Cardiff (19501965) sans changement majeur des voies, et constructions neuves en béton adaptées au site pour le Diesel à Laira, région ouest des BR près Bristol (1962). Dessins Y. Machefert-Tassin d'après des documents fournis par les réseaux concernés. 
Ainsi, en Europe, en dehors de la France, on constate la prédominance des installations rectangulaires à voies parallèles, remises et ateliers inclus, avec des proportions décroissantes. À titre d'exemple : Hollande $(90 \%)$, Belgique $(80 \%)$, Allemagne $(70 \%)$ mais aussi l'Italie $(70 \%)$ et, à des degrés moindres, l'Espagne $(50 \%)$, l'Autriche $(60 \%)$ et les autres... (Russie comprise).

En dehors de cet aspect d'architecture des remises, très différenciée heureusement d'un pays, voire d'une région ou d'un réseau à l'autre, mais sur des schémas de voies classiques, on trouve également les autres installations, liées aux voies, et sur lesquelles s'effectuent la visite, le nettoyage, l'approvisionnement, l'orientation et le classement des engins de traction, du moins pour les locomotives ou motrices isolées des rames. Leurs installations sont linéaires ou ponctuelles et souvent copiées d'un réseau à l'autre, donc facilement adaptables ou transformables, à l'exception de la décharge directe du charbon depuis les wagons sur voie ou en remblai ou estacade aux tenders en contrebas, chers à la Grande-Bretagne et a ses dominions - les autres «montent » le charbon en stock. Un exception généralisable : les grands silos à charbons et les parcs à combustibles correspondants, spécifiques des plus grands dépôts vapeur. Il est certain que les besoins, très différents selon les modes de traction, vapeur, électrique, autorails ou Diesel, comportent des éléments communs standardisables (visite, graissage, sablage par exemple) qui conduisent, après 1930, soit à la spécialisation d'établissements, que l'on constate encore de nos jours en Europe, soit, pour ceux ayant une vocation mixte, à la juxtaposition inévitable de faisceaux de voies spécialisés (fig. 3 et fig. 10).

Si l'on revient à la chronologie, on peut dire, schématiquement, et ceci est valable pour l'ensemble de l'Europe, que les dépôts à modes de traction mixtes ont comporté les adaptations additionnelles suivantes :

- en général la mixité par la succession vapeur $>$ autorails $>$ locomotives Diesel (fig. 3 et fig. 11)

- souvent, le passage vapeur > électricité > autorails ou locomotives Diesel (fig. 5 et fig. 12)

- plus rarement, électrique seul (fig. 8) ou autorails seuls, puis éventuellement ajout de locomotives Diesel (voir RHCF 18)

- enfin, depuis les années 1980, le développement des banlieues électrifiées, en rames réversibles ou en automotrices puis les Dieselélectriques de ligne (TEE ou HST, High Speed Trains) et surtout l'apparition des rames électriques à grande vitesse homogènes et en grand nombre ont conduit au développement nouveau d'installations 
spécifiques souvent situées à l'emplacement de faisceaux de garage ou d'ateliers d'entretien des voitures et non des dépôts (France, GrandeBretagne). Certaines installations sont juxtaposées à des dépôts d'engins moteurs (Belgique, Allemagne, Italie) dont les bâtiments et les voies existantes conservent leur activité propre (ex. Hambourg). D’autres enfin (Italie, Grande-Bretagne) sont totalement neuves et établies sur d'autres lieux.

Les installations européennes nouvelles ne différent alors généralement entre elles que par des détails et non par les dispositions essentielles (faisceaux de voies et remises en long). Il en est ainsi, en dehors de la France, en Grande-Bretagne (dépôts HST puis Eurostar à North-Pole), Belgique (Forest) et surtout Allemagne (ICE) à Hambourg (fig. 13) puis à Munich et Berlin, Italie (Milan, Rome) et aussi l'Espagne ( $A V E$ et Talgo) (Madrid-Sud à la Sagra). Et nous ne pouvons que nous réjouir de voir se multiplier ces nouvelles installations, souvent de même style, à voies parallèles sous abris très longs de 400 à $800 \mathrm{~m}$. Elles représentent une forme, certes récente mais déjà aboutie, des dépôts pour trains entiers d'aujourd'hui (voir fig. 3 et 13). Seuls y ont changé les gestes du métier des hommes, mais non leur savoir-faire. Certes les bâtiments et les machines outils ont dû être adaptés, les remises à voies parallèles étant sur fosses à pilotis, avec des machines spécialement conçues : tours en fosse après examen des roues à chaque rentrée avec enregistrement des profils, par exemple, de même que des moyens à grande rapidité d'intervention, levage simultané de plusieurs caisses sur vérins synchronisés et échange standard de blocs entiers d'appareils soit par le haut (ponts roulants comme précédemment) ou par le bas (chariots autonomes de mise en place ou retrait). Ces appareils de manutention nouveaux doivent se compléter d'ateliers très spécifiques et de vastes magasins mécanisés pour le remplacement et le transfert d'organes avant réparation ailleurs.

En outre, concernant le savoir-faire déjà mentionné, l'introduction des éléments informatiques, jusque dans des ateliers ou bâtiments parfois presque centenaires, a évidemment bouleversé les habitudes et nécessité de nouveaux comportements, de nouveaux gestes avec souvent beaucoup plus de technologie dans l'usage du système, mais surtout de connaissances et compétences techniques dans de nombreux domaines d'analyse préventive, de préférence à la réparation corrective. Ceci est universel dans tous les établissements dits aujourd'hui "industriels », concernant la maintenance du matériel roulant et pas seulement des locomotives, automotrices ou autorails. C'est là sans doute que, beaucoup plus encore que dans les installations qui s'accommodent souvent de bâtiments existants, réside une évolution radicale (fig. 14). 


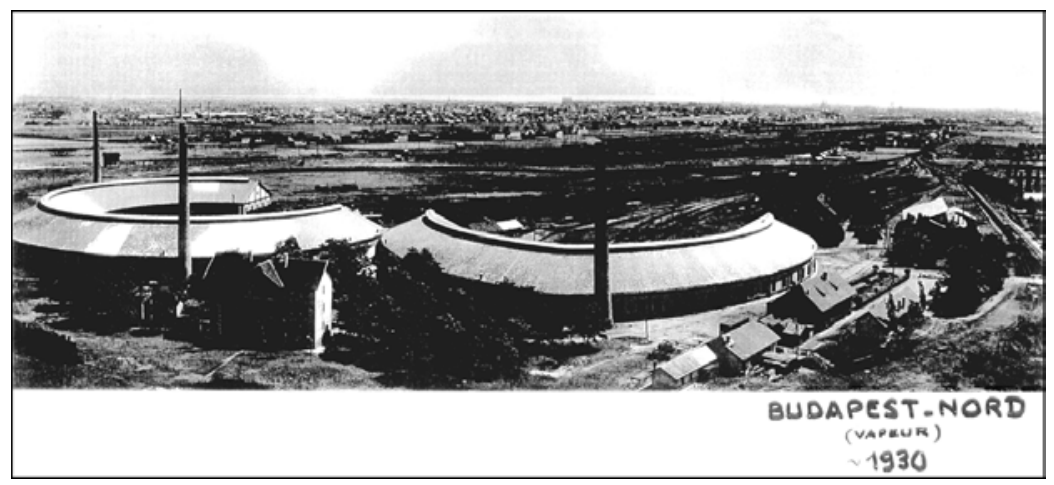

Figure 12. Même en Europe centrale, vers 1930, on trouve encore comme à Budapest-Nord des rotondes annulaires de style très ouest ou sud-ouest français. On notera, comme en Allemagne, le souci écologique, dirionsnous aujourd'hui, de la collecte des fumées vers les hautes cheminées de brique essayées en France par le Nord à La Chapelle et à Calais vers 1930. Cl. MAV, coll. Y. Machefert-Tassin.

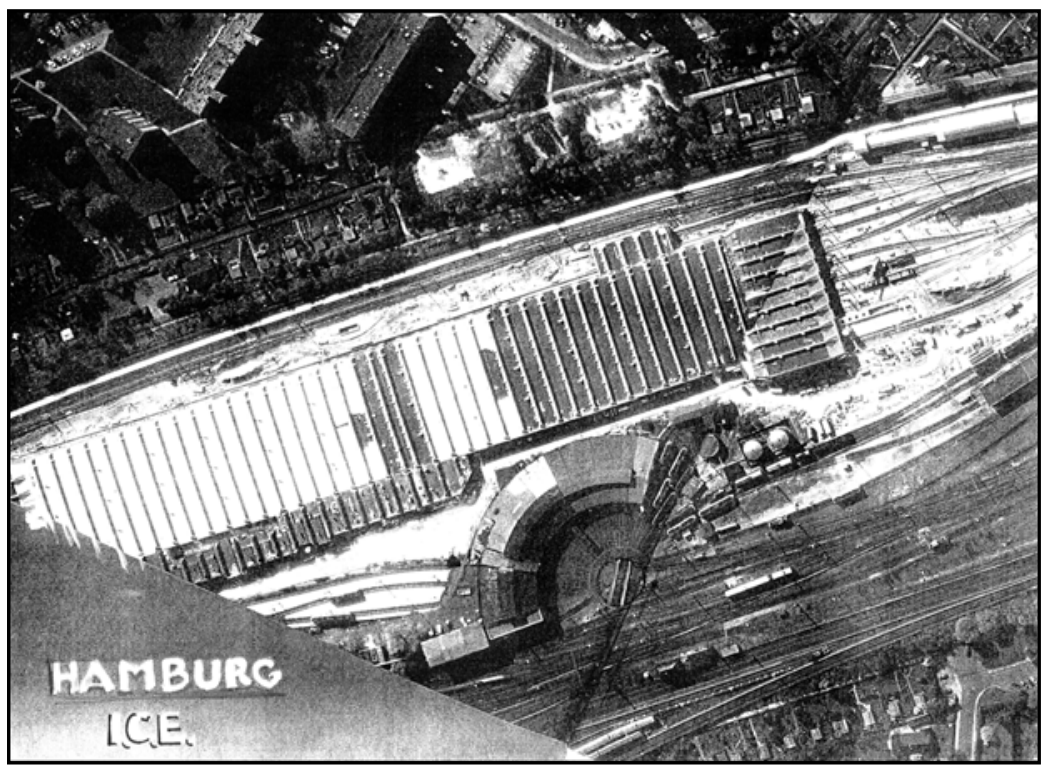

Figure 13. Vue aérienne à Hambourg (1990) du premier atelier et dépôt allemand pour rames à grande vitesse ICE. On notera qu'il intègre l'ancien dépôt vapeur devenu électrique et Diesel, pour locomotives, d'AltonaNord (situé à Eidelstedt). Cl. Deutsche Bahn Ag., coll. Y. Machefert-Tassin. 


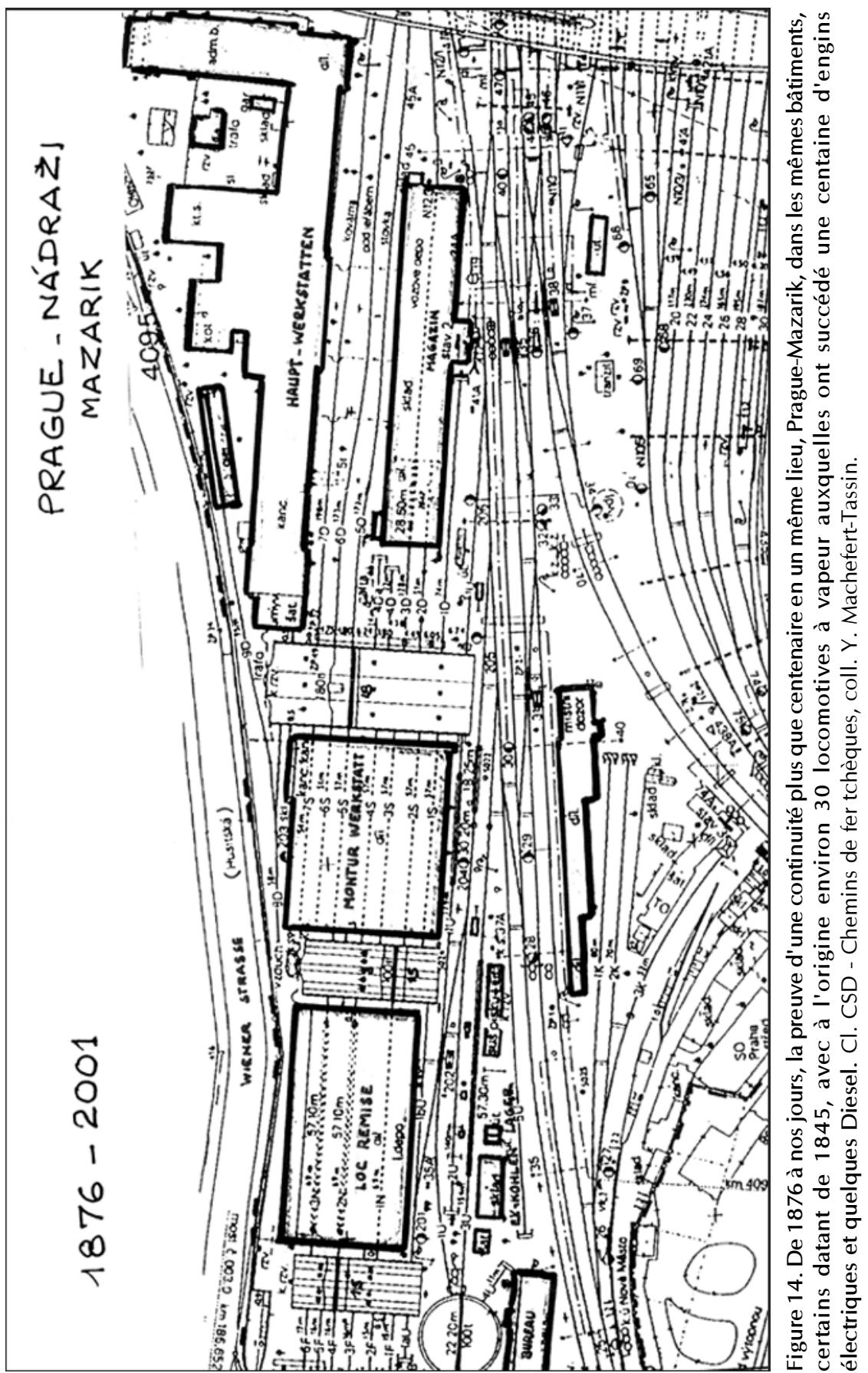


En particulier, citons les approvisionnements, visite et entretien intégrés désormais à la maintenance générale, de plus en plus préventive que réparatrice, sur des trains complets, avec des gains certains en maind'œuvre spécialisée et surtout en temps passé, expliquant l'usage des longues remises à voies parallèles d'aujourd'hui, côtoyant parfois les rotondes à rayons individualisés et multiples d'hier. Nous en verrons de multiples exemples au cours de cette journée. 